\title{
MERCOSUR y UNASUR: Una aproximación al tratamiento de la movilidad de personas ${ }^{1}$
}

\author{
MERCOSUR and UNASUR: an approach to the treatment of human mobility
}

\author{
Lucila Nejamkis Rubellin \\ Consejo Nacional de investigaciones Científicas y Tecnológicas, Argentina, \\ Inejamkis@unaj.edu.ar
}

Fecha de envío: 24/08/2016. Fechas de reenvíos: 29/11/2016 - 1/12/2016, 7/02/2017 - 8/03/2017, Fecha de aprobación: 19/04/2017. Fecha de publicación: 18/05/2017.

Resumen: La integración regional constituye un proceso complejo que involucra un conjunto de fenómenos políticos, económicos, culturales y sociales sobre los cuales los estados deben lograr acuerdos a fin de definir políticas coordinadas. El tránsito de personas y la regulación de los flujos migratorios forman parte de estos aspectos que por abordar. En los últimos años, los diferentes bloques regionales de América del Sur (MERCOSUR, CAN, UNASUR) han pretendido avanzar en esta dirección. Conceptos como la libre circulación y la ciudadanía regional han dominado las propuestas, aunque no siempre con éxito. Este proceso se ve acompañado por transformaciones de las políticas migratorias a nivel local. Argentina como pionero, en el año 2003 abrió un espacio de discusión, seguido por Uruguay y Perú que también modificaron sus normativas. Por su parte Brasil, Paraguay, Chile y Ecuador, se encuentran en un proceso de debate acerca de la necesidad de reformar su legislación migratoria. Los propósitos de este trabajo son: (1) analizar el vínculo entre proceso de integración regional y políticas migratorias, específicamente en el MERCOSUR y UNASUR entre los años 2002 y 2014, y (2) indagar cómo influye la regionalización en las decisiones nacionales vinculadas a la temática migratoria.

Palabras claves: integración regional; migraciones; políticas públicas; Sudamérica.

\begin{abstract}
Regional integration is a complex process that involves a set of political, economic, cultural and social phenomena in which State members must reach agreements to define coordinated policies. The movement of people and the regulation of migration flows are some of the aspects to be addressed. In recent years the different intergovernmental regional organizations of South America (MERCOSUR, CAN, UNASUR) have tried to move in this direction. Concepts such as freedom of movement and regional citizenship have led the proposals, although not always successfully. This process is accompanied by changes in local immigration policies. Argentina, as a pioneer in 2003, opened an area for discussion, followed by Uruguay and Peru who also amended their regulations. In turn, Brazil, Paraguay, Chile, and Ecuador
\end{abstract}


are discussing the need to reform their immigration legislation. This paper is aimed at (1) analyzing the link between regional integration process and immigration policies, specifically in MERCOSUR and UNASUR between 2002 and 2014 and (2) finding out how regionalization influences national immigration decisions.

Keywords: Regional Integration; Migration; Public Policy; South America

\section{Introducción}

La integración regional constituye un proceso complejo que involucra un conjunto de fenómenos políticos, económicos, culturales y sociales sobre los cuales los estados deben lograr acuerdos a fin de definir políticas coordinadas. El tránsito de personas y la regulación de los flujos migratorios forman parte de estos aspectos a ser abordados.

En los últimos años los diferentes bloques de América del Sur (MERCOSUR, CAN, UNASUR), han intentado avanzar en esta dirección. Conceptos como la libre circulación y la ciudadanía regional han dominado las propuestas, aunque no siempre se han logrado los objetivos que estas nociones suponen. Este proceso se ve acompañado por transformaciones de las políticas migratorias a nivel local. Argentina, como pionera, en el año 2003 abrió un espacio de discusión, seguido por Uruguay, quien también modificó su normativa en 2008. Por su parte Bolivia lo hizo en el año 2013, mientras que Perú en el 2015. Actualmente, tanto Brasil (que en diciembre de 2016 aprobó la ley en la Cámara de Diputados, y solo falta que se haga en la de senadores), como Chile, Ecuador y Paraguay, aunque este último en menor medida, se encuentran en un proceso de debate acerca de la necesidad de reformar sus legislaciones migratorias. Estas modificaciones permiten dar cuenta del reconocimiento a escala regional de la necesidad de homologar el tratamiento multilateral del fenómeno migratorio con las políticas sobre migración históricamente asociadas a la administración interna de cada estado.

En relación con lo anterior, los elementos más ambiciosos que la integración regional plantea en materia de migraciones son: el proyecto de decisión para la incorporación de la Conferencia Sudamericana sobre Migraciones en la estructura de la UNASUR y el establecimiento de una hoja de ruta que permita construir la ciudadanía sudamericana (UNASUR, 2012). Si bien se trabajará a lo largo del texto, sobre los avances y retrocesos en esta dirección, es dable aclarar que estas propuestas implicaron un proceso intenso de trabajo al interior del bloque. Esto encuentra relación, tal como explica Novick, con las múltiples dificultades en los procesos de negociación. Es importante entender que, dentro de cada uno de los países, la necesidad de internalizar los acuerdos logrados muestra dos tendencias: por un lado genera tensiones entre los poderes ejecutivos y los poderes legislativos, basadas en los diferentes sectores sociales e intereses que cada uno de ellos representa; $y$, por el otro, motiva dilaciones que ponen en duda la efectividad de la integración misma (Novick, 2012). 
En este contexto, el propósito de nuestro trabajo consiste en analizar el vínculo entre el proceso de integración y la normativa en torno a la movilidad de personas a nivel regional, específicamente en el MERCOSUR y la UNASUR. El trabajo tiene como objetivo reflexionar, entre otras cosas, sobre: ¿Qué relaciones se establecen entre las migraciones, las políticas migratorias y el proceso de integración regional sudamericano? Para responder a estos interrogantes trabajaremos, en primer lugar, con un conjunto de elementos teóricos que nos permitirán reflexionar sobre las reconfiguraciones estatales que se establecen a partir de la década de 1970 a nivel mundial, cómo esto repercute en los procesos de integración regional y su vinculación con el tratamiento del fenómeno migratorio. En segundo lugar, nos proponemos profundizar el estudio de caso de MERCOSUR y UNASUR para, con base en experiencias concretas, dar cuenta de las especificidades de esta problemática.

A través del estudio de caso, y sobre la base de la problemática y los objetivos propuestos, la metodología de investigación consiste en el uso integrado y complementario de enfoques y herramientas de análisis cualitativo como es el análisis documental y el trabajo con fuentes secundarias. Desde esta perspectiva, se utiliza el análisis sociojurídico y documental de la normativa (planes, acuerdos, declaraciones, etc.) (García Gutiérrez, 2002; Iglesias y Gómez, 2004). Las normas regulatorias, aunque resulta evidente que se encuentran comprendidas en aquello definido como "políticas públicas", cristalizan de manera discursiva o expresan de manera manifiesta el objeto o el fin de las políticas que pretenden atender. Así que, metodológicamente, las normativas aparecen como la "puerta de entrada" para el estudio de las políticas, en tanto se encuentran comprendidas en un cuerpo documental que reviste coherencia y exhaustividad (García Gutiérrez, 2002).

Se propone recortar el análisis al año 2014, ya que a partir de 2015 los procesos de integración en la región se encuentran plagados de incertidumbre, debido a los cambios de signo político de gobiernos de peso, para los distintos bloques, como son Argentina y Brasil. En este contexto, el tratamiento a la movilidad de personas en el territorio se vuelve, por momentos, una incógnita que por la contemporaneidad de la temática se encuentra fuera del análisis de este trabajo.

\section{Herramientas teóricas para pensar las políticas migratorias en los contextos de integración regional}

Pensar las políticas migratorias desde los procesos de integración, o los procesos de integración desde las políticas migratorias implica reconocer las lógicas estatales (nacionales), a través de las cuales se han elaborado la políticas públicas sobre migración a lo largo de los años, a la vez que requiere introducir nuevos elementos vinculados a la interestatalidad o la supranacionalidad, dependiendo de cuál sea la base de los acuerdos de integración firmados por los distintos bloques regionales. Por consiguiente, cuando se propone indagar acerca de la relación entre las migraciones, las políticas migratorias y los procesos de integración, es necesario conjugar 
miradas locales y globales para comprender la intersección de las formas de funcionamiento estatales y las transformaciones que estas han tenido a raíz de su participación en un proceso de integración regional (De Sierra, 2001; Mellado, 2009).

El debate teórico sobre el control de los flujos se ha centrado, en los últimos años, en la erosión de la soberanía del Estado por los efectos de la globalización y la aparición de otros actores supranacionales, así como también por la importancia de los vínculos entre las relaciones transnacionales y las políticas (Meyer, 2006). En esta línea de análisis, algunos trabajos se han basado en el impacto de la migración en las relaciones internacionales y, específicamente, en el vínculo entre migración, seguridad nacional y políticas migratorias (Teitelbaum y Weiner, 1995, citados en Meyer, 2006). Empero, se ha criticado que estas teorías se preocuparon más por la cuestión de la seguridad que por los temas sociales que involucran a las minorías de origen migratorio.

Se reconoce que el inicio de la década de 1970 ha marcado el comienzo de una profunda reestructuración económica y social de alcance mundial, con efectos sobre la organización de los mercados, el desarrollo tecnológico, la deslocalización industrial, cambios en la organización del trabajo asalariado -con el consiguiente aumento de la desocupación y precarización laboral-, etc. Estos cambios conllevan también modificaciones en la forma de territorialidad. Para dar cuenta de ello, establecen el ejemplo de la Unión Europea, entendiendo que esta misma no se constituyó como territorio hasta el advenimiento de las políticas de control migratorio común (Walters y Haahr, 2005).

Las reconfiguraciones estatales generadas a partir de los procesos de integración regional han hecho repensar los alcances del concepto de soberanía. En este contexto, han proliferado las discusiones sobre la pérdida de soberanía de los Estados nacionales. En relación con lo anterior, Saskia Sassen señala que la soberanía "sigue siendo una propiedad sistémica", aunque "su inserción institucional y su capacidad para legitimar y absorber todo el poder legitimador, es decir, para ser la fuente de la Ley, se han tornado inestables" (Sassen en Mezzadra, 2012, p. 169). El poder se está reconfigurando, en un contexto en el que la lógica de la soberanía se entrelaza con la lógica de la gobernabilidad neoliberal, con una gobernanza que se presenta como un sencillo proceso de persuasión, sin coerción, según patrones neutros de cálculo y gestión de riesgos. Los propios sujetos de soberanía son cada vez más cambiantes y heterogéneos. El régimen migratorio global en desarrollo constituye un claro ejemplo al respecto: se trata de un régimen del ejercicio de la soberanía con una estructura híbrida y mixta (Mezzadra, 2012). Este tipo de análisis permite evidenciar la contradicción entre una necesidad cada vez mayor de articular los espacios nacional, regional e internacional, a la vez que dar cuenta como la noción de soberanía de los estados nacionales ha cumplido y sigue cumpliendo un rol fundamental al momento de definir las políticas públicas en torno al movimiento de personas. 
Por consiguiente, si bien sirve de "guía" el preguntarnos por los usos de la frontera en los procesos de integración regional, reconocemos los riesgos y las implicaciones de transpolar otras experiencias, como puede ser la europea a nuestra región. Las experiencias migratorias regionales son disimiles, las líneas divisorias entre norte y sur, países desarrollados y en vías de desarrollo, generan distintos procesos de circulación y diferentes respuestas estatales y regionales que deben ser atendidos teniendo en cuenta la especificidad de cada caso.

En este sentido, tal como plantea Grimson (2005), los estudios sobre las fronteras de Europa, África y Asia indicaban que las fronteras del mundo son muy heterogéneas e irreductibles las unas a las otras. No solo son diversas las relaciones interestatales, sino también los vínculos entre las sociedades fronterizas y sus estados nacionales.

Atendiendo a nuestro objeto de estudio, el caso sudamericano, resulta importante remarcar, tal como plantea Machado de Oliveira, que, por ejemplo, en el MERCOSUR no hay frontera, hay fronteras. La integración regional no pone fin a las fronteras ni a las interacciones en frontera, solo las redimensiona, haciéndolas más visibles (por estimularlas o limitarlas) dando lugar a una lucha entre el reconocimiento de los factores idiosincrásicos -por un lado- y la necesidad de propender a las generalidades por el otro (Machado de Oliveira, en Rhi Sausi y Conato, 2009).

\section{Los casos de MERCOSUR Y UNASUR}

A lo largo del siglo XX han sido numerosas las propuestas de integración que ha habido en el Cono Sur: el Tratado ABC de 1915, el intento por reestablecer dicho tratado en 1953, la Asociación Latinoamericana de Libre Comercio en 1960 y la Asociación Latinoamericana de Integración en 1980 constituyen los ejemplos más destacables (Carrera, 2005). Estos acuerdos no han sido mayormente exitosos y sus logros concretos fueron escasos. En el año 1991, a partir de la firma del tratado de Asunción por parte de Argentina, Brasil, Paraguay y Uruguay (que crea MERCOSUR) ${ }^{2}$, la integración, en sí misma, deja de ser discutida y se genera un importante consenso entre las distintas fuerzas políticas y sociales de los países miembros del bloque acerca de la importancia de consolidar la integración regional (Novick, 2010). El personal experto puntualiza que el contexto en que nace el Mercosur se caracteriza por la coincidencia en la aplicación de programas de apertura, liberalización y desregulación; y por el fuerte presidencialismo en las relaciones bilaterales y multilaterales regionales, rasgo que influiría en el formato institucional en que quedó plasmado (Bernal-Meza, 2000).

En cuanto a las especificidades de los contextos de integración que abordamos en este trabajo, resulta fundamental destacar las diferentes tramas históricas en las que estos procesos se han iniciado. Por un lado, el momento histórico de surgimiento del MERCOSUR, creado en la década

2 Integrado por Argentina, Brasil, Paraguay, Uruguay y Venezuela. Tiene como países asociados a Bolivia, Chile, Colombia, Ecuador y Perú. 
de 1990, en pleno auge de la implementación de políticas neoliberales en el Cono Sur y, por el otro lado, el escenario regional que resultó en la fundación de la UNASUR3 ${ }^{3}$, originada bajo el predominio de gobiernos que se acercaban a una lógica que podría denominarse posneoliberal $4{ }^{4}$ (Sader, 2008), que ha implicado otra manera de abordar la problemática migratoria por parte de los Estados. Entendemos que, a partir de los años 2000, las transformaciones en la manera de entender lo migratorio se encuentran asociadas a los cambios propuestos por gran parte de los gobiernos de la región en los planos políticos, económicos, y sociales que posibilitaron un modelo de integración distinto al que marcaron los años 90 . Este nuevo modelo da a la integración latinoamericana un lugar prioritario como proyecto político regional y como alianza estratégica.

Tal como explican Bono y Bogado Bordazar (2014), el MERCOSUR y la UNASUR han surgido a la vida jurídica y política internacional con fundamentos teóricos e históricos diferentes, lo cual ha denotado la construcción de modelos regionales específicos para cada caso. En parte el surgimiento de la UNASUR responde a la necesidad de incentivar y profundizar los procesos de integración desde una óptica diferente a la comercial, especialmente vinculada a la vida política y social. Fue a partir de allí que los diferentes Estados -a lo largo de toda la décadahan impulsado desde distintas instituciones nacionales y regionales, nuevas estrategias para reorientar el modelo de integración y cooperación, que ya estaba dando señales de decadencia.

Es importante destacar que los diferentes países que integran el bloque regional presentan distintas realidades económicas, políticas, sociales, etc., que resultan en disímiles experiencias migratorias. Los países de mayor atracción de población dentro de la UNASUR son Argentina y Venezuela, mientras que los de mayor expulsión son Colombia y Paraguay (CEPAL). Esto, a su vez, se traduce en disimiles políticas migratorias y preocupaciones de los Estados.

En el caso particular de Sudamérica, a partir de la creación del Mercosur, múltiples trabajos se han dedicado a indagar sobre los cambios acaecidos en el tratamiento de la cuestión migratoria en la región; se toma como referencias, entre otros, a los trabajos de Marmora (2010), Novick (2005), Domenech (2007), Perez Vicich (2000), Maguid (1999). Estos estudios coinciden en que, a partir de la década de 1990, nuevos actores comienzan a intervenir en el debate migratorio y en la definición de políticas, estableciéndose una articulación cada vez mayor entre los espacios nacional, regional e internacional en su tratamiento político (Domenech, 2007). De hecho, algunos especialistas han comenzado a hablar de la emergencia de una nueva tendencia hacia

3 Antes conocida como Comunidad Sudamericana de Naciones, se encuentra conformada por la totalidad de los países de América del Sur. Algunos Estados, a su vez, forman parte de procesos de integración: el Mercado Común del Sur (Argentina, Bolivia, Brasil, Chile, Colombia, Ecuador, Paraguay, Perú, Uruguay y Venezuela) y la Comunidad Andina (Bolivia, Ecuador, Colombia, Perú).

4 Tal como explica Sader (2008), esta es una categoría descriptiva que designa diferentes grados de negación del modelo anterior, sin llegar a configurar uno nuevo: en líneas generales, esto implica un cuestionamiento a la "bondad del mercado" como único asignador de recursos, así como expresa correlaciones de fuerzas sociales más favorables al acotamiento del poder del capital global, y también recupera resortes estatales para la construcción política. 
la internacionalización o regionalización de las políticas migratorias en la región (López Sala citado en Nicolao, 2015). De acuerdo con el alcance de este tipo de marcos cooperativos, suele hablarse de internacionalización", en tanto concepto más genérico, como de "regionalización" de las políticas migratorias, para hacer referencia a espacios de cooperación circunscriptos a una región geográfica, y vinculados a proyectos integracionistas. En la misma línea otras autorías afirman que, con una dinámica lenta, pero sostenida, el proceso de integración influyó ciertamente en la definición de las políticas nacionales, de algún modo "desnacionalizándolas" (Novick, 2010).

\section{Procesos regionales}

Este apartado permitirá dar cuenta de que, si bien ya existían, a partir del desarrollo de los procesos de integración regional, han crecido los instrumentos bilaterales, regionales y multilaterales para el tratamiento de las migraciones. En relación con lo anterior, es importante entender cómo estas transformaciones impactaron en la manera de "gestionar" las migraciones en la región. En los últimos años son múltiples las iniciativas bilaterales que promueven mecanismos intermedios para la solución de problemas internos y transfronterizos en materia migratoria. La proliferación de estos acuerdos es interpretada por algunos autores como una forma de remediar la falta de un consenso efectivo por parte de los Estados miembros del MERCOSUR y asociados, que no han encontrado punto de encuentro en temas prioritarios. Solo por mostrar algunos ejemplos: Iniciativas de regularización ciudadana entre Bolivia- Brasil (2005, 2006, 2007), Argentina- Brasil (2005), Brasil- Uruguay (2002, 2006), Paraguay y Brasil, así como los procesos de regularización implementados por el gobierno chileno $(2007,2008)$, la regularización migratoria que Brasil llevo a cabo en el año 2009; todo ello se podría citar, entre muchos otros, como esfuerzos importantes que buscan resolver con urgencia el tema de la libre circulación (Mondol Lopez, 2010).

En otras investigaciones analizamos cómo, a partir de principios del siglo XXI, el abandono de la perspectiva neoliberal y el establecimiento de un cambio de rumbo basado en el predominio de la integración latinoamericana, en términos de política interna y externa, repercutieron de forma positiva en el tratamiento de las políticas regionales de movilidad de personas (Nejamkis, 2016). Cuando se hace referencia a una connotación positiva en la forma de concebir el fenómeno migratorio, entendemos, en primer lugar, que se colocó el tema en la agenda regional, y que se fueron generando espacios y resoluciones que abogan por mayores derechos de los grupos migrantes, tanto en el sentido del ingreso y la regulación como en la ampliación del acceso a derechos (entre estos se encuentran la conferencia Sudamericana de Migraciones, y el Foro especializado Migratorio). Igualmente es importante tener en cuenta aquellas perspectivas que cuestionan los verdaderos intereses que se encontrarían detrás de este proceso, entendiendo que bajo la cobertura ideológica de esta nueva perspectiva técnico política se desarrolla una política de control con rostro humano. Tal como explica Eduardo Domench, este control sobre la 
inmigración irregular desplaza, sin eliminar, formas restrictivas o coercitivas de acción, pero no con el propósito de hacer efectivos los derechos humanos de los migrantes, sino para obtener mayores resultados en el control de los flujos (Domenech, 2013, p. 2).

En primer lugar, los pasos más contundentes en términos de posicionamientos a nivel regional los dieron las declaraciones de Lima y Buenos Aires. En el año 1999 el Encuentro Sudamericano sobre Migraciones, Integración y Desarrollo realizado en Lima convocó a Argentina, Bolivia, Brasil, Colombia, Chile, Ecuador, Paraguay, Perú, Uruguay, y Venezuela. De este primer encuentro surgió la Declaración de Lima que reconoce los estrechos vínculos de las migraciones con los problemas de desarrollo económico y social de los países involucrados. En esta línea, el documento da el puntapié inicial para que los países salgan de su órbita individual y amplíen su análisis al contexto regional, continental y mundial. Otro dato de importancia en el avance del tratamiento de la temática migratoria en la región es haber dado cuenta que el respeto de los derechos humanos de los migrantes constituye una preocupación básica para los países. En resumen, este documento resalta la "respuesta asociativa e interactiva a través de los procesos de integración subregional como son el MERCOSUR y la Comunidad Andina, dentro de los cuales se están realizando avances significativos en el tratamiento de las migraciones" (Di Filippo, 2000, p.10).

Como seguimiento a este primer encuentro, en el año 2000, se celebró la Conferencia Sudamericana sobre Migraciones con los mismos participantes a los cuales se sumaron Guyana y Surinam. De aquí emana la Declaración de Buenos Aires, la cual en sus considerandos avanza en la misma línea que la declaración anterior y resalta la necesidad de "abordar la problemática migratoria de la región, a través de mecanismos de diálogo multilateral abierto que promuevan la reflexión y la coordinación de políticas y acciones entre países que comparten esta problemática, mediante el ejercicio pleno de la cooperación regional para fortalecer los actuales procesos de integración y desarrollo que se dan en la región" (Di Filippo, 2000, p.1). A su vez, vuelve a poner en agenda el tema de los Derechos Humanos como preocupación fundamental. Si bien lo anterior es importante, se debe destacar que en ese mismo punto aplican los lineamientos de la Organización Internacional para las Migraciones al proponer la promoción de migración ordenada y planificada como solución a la migración irregular y la lucha contra el tráfico de seres humanos. En materia de puesta en práctica de las políticas propuestas, la declaración, entiende que es necesario el fortalecimiento institucional de los organismos nacionales, la capacitación del personal de instituciones públicas, la actualización de las legislaciones nacionales y el desarrollo de mecanismos de coordinación entre los Estados participantes. Para dar continuidad a estos dos primeros eventos, en la parte resolutoria de la Declaración los representantes deciden constituir "un Foro de coordinación y consulta en materia migratoria para los países de América del Sur". El objetivo del Foro será "un tratamiento integral de la temática migratoria, sin perjuicio de los demás mecanismos análogos de integración subregionales y concertación bilateral" (Di Filippo, 2000, p.11). 
Por su parte, el Foro Especializado Migratorio (FEM), del 2003, es un espacio que funciona en el ámbito de las Reuniones de Ministros de Interior del MERCOSUR y Estados Asociados, y tiene entre sus funciones el estudio del impacto de las migraciones, en la región y fuera de ella, y el análisis y desarrollo de proyectos de normas o acuerdos en materia migratoria que regirán para los países del bloque. Según lo analizado, se puede decir que este es el primer espacio en la historia del MERCOSUR concentrado exclusivamente en el tratamiento de esta problemática. Uno de los primeros documentos que lanzó el FEM fue la Declaración de Santiago de Principios Migratorios (2004), en la cual se plasma el espíritu con el que los gobiernos se comprometen a encarar la política migratoria. Se desprende del análisis de este documento una serie de avances hacia los que ha ido el tratamiento migratorio en la región. Entre los elementos más novedosos que no habían sido trabajados en las declaraciones anteriores se destacan: a) El reconocimiento al aporte de migrantes en la formación de los Estados; b) La reafirmación de los compromisos que los Estados tienen de brindar y promover la protección internacional a las personas refugiadas, tal como está previsto en la Convención de Ginebra de 1951 y su Protocolo Complementario de 1967, y otros instrumentos internacionales sobre la materia a los cuales los entes declarantes se adhirieran en el futuro; c) Requerir a los países extra-bloque otorgar un tratamiento justo y humanitario a los grupos inmigrantes de nuestra región, en correspondencia con el trato brindado a sus nacionales en nuestros territorios; d) Se destaca la importancia de la reunificación familiar como elemento necesario para la estabilidad plena de los grupos inmigrantes, reconociendo a la familia como base fundamental de la sociedad; e) Se reconoce el derecho de los estados de ejercer el adecuado control de sus fronteras, pero sin tratar la irregularidad migratoria como hecho punible de derecho penal; f) Se reafirma el compromiso de combatir al tráfico ilícito de migrantes, la trata de personas, el tráfico de personas menores y otras formas de delitos transnacionales, entre otros temas.

Otro de los elementos de gestión que ha emanado de la Décima Conferencia Sudamericana de Migraciones es el Plan de Desarrollo Humano para las Migraciones del año 2010. Se cuentan como principios rectores del plan: el respeto de los derechos humanos de las personas migrantes; el ejercicio ciudadano de la libre movilidad; la garantía de la ciudadanía plena para las personas migrantes, que implica la participación ciudadana, el acceso a las diversas áreas sociales y la participación cultural; la coherencia nacional y regional de las políticas migratorias; el reconocimiento de los efectos positivos de la migración; y el reconocimiento de las personas migrantes como parte de los procesos de integración regional (Martínez Pizarro, 2011). En relación con lo anterior, uno de los principales avances es la propuesta de comprender el hecho migratorio desde su integralidad, es decir, considerar todos los niveles que abarca este fenómeno que también se debe articular con las instituciones y administraciones regionales y estatales. Dentro de los lineamientos estratégicos del plan, uno de los elementos principales para pensar los avances en materia regional es el planteo de la necesidad de una coherencia normativa que implica contemplar dos planos: el de la normativa interna de cada país, y el de la externa a la región. 
Tal como se ha mencionado previamente, el rol de la Organización Internacional para las Migraciones ha sido primordial en las propuestas regionales. En el caso del Plan de Desarrollo Humano para las Migraciones se observa claramente cómo uno de los principales impulsores ha sido la OIM, cuyos intereses están vinculados a "controlar"la inmigración en la región. En este sentido, se observa que dentro de los lineamientos propuestos se plantea el fortalecimiento de la "gestión" migratoria -que según el plan es la herramienta básica para la ejecución de políticas y programas que aseguren los principios del irrestricto respeto por los derechos humanos de las personas migrantes, y el principio del ejercicio ciudadano a la libre movilidad- abarca varias áreas a tratar: la coordinación interinstitucional nacional e interregional; la atención de la movilidad humana en áreas de frontera, y la capacitación de los agentes migratorios.

En este sentido, tal como viene planteando la OIM desde hace varias décadas, se propone pasar de la "regulación" de los flujos en los países de destino al "control" de los flujos en los países de origen. La idea de gestión que proviene de la adaptación dellenguaje del management (Estupiñan Serrano, 2013), planea hacer las migraciones "dirigibles", "canalizables" y "aprovechables" (Marmora, 2010). Ahora bien, uno de los principales problemas de esta visión es que esta Organización "no cuestiona, ni pone en entredicho el enfoque, ni las relaciones asimétricas, de dominación y de poder que se dan a nivel global" (Ramírez y Alfaro, 2010 p. 9). En este sentido, multiplica las mismas fórmulas para regiones diferentes casi como una estrategia de marketing que poco tiene que ver con mejorar las condiciones de vida de la población migrante.

En relación con el Plan de Desarrollo Humano para las migraciones, si bien lo anterior muestra un gran avance en tanto declaración de principios, algunos autores entienden que si se atiende al listado de acuerdos y compromisos negociados por el Foro Especializado Migratorio desde su creación, claramente ninguno supera en términos de impacto y trascendencia el Acuerdo de Residencia para Nacionales de los Estados Partes del MERCOSUR, pues en su gran mayoría están volcados a compromisos relacionados con algún aspecto administrativo, técnico o burocrático (documentos de viaje, bases de datos compartidas, legalizaciones, entre otras), respecto de la movilidad intrarregional (Nicolao, 2015).

En este sentido, se evidencia que los más sobresalientes de este proceso se encuentran en una serie de acuerdos emanados de la XXII Reunión de Ministros del Interior del MERCOSUR, Bolivia y Chile (2002), donde se observa un cambio de orientación en el tratamiento de la cuestión migratoria en la región. En dicho encuentro se aprobó el Acuerdo de Regularización Migratoria y el Acuerdo sobre Residencia para Nacionales de los Estados Partes del MERCOSUR, los cuales constituyen un salto cualitativo en tanto se comienza a plantear la integración de la región desde una óptica que "reivindica la condición de ciudadano del MERCOSUR para garantizar la residencia legal en cualquiera de los países que lo componen" (Maguid, 2007, p.242), y se vuelve a colocar al concepto de libre circulación -pensando ahora sí en una movilidad de asentamientoen el centro del tratamiento de los flujos migratorios en el espacio de integración. 
Por su parte, Arcazo y Freire (2015) entienden que la adopción del Acuerdo de Residencia ha de comprenderse también dentro del contexto de crisis política que se vivió por parte del Mercosur con el cambio de siglo y la necesidad de introducir nuevos elementos en la agenda regional para relanzar el proceso de integración. Autores como Margheritis han mencionado asimismo la competencia ideológica por el liderazgo post-neo liberal entre Brasil y Argentina y la importancia de la propuesta argentina para un acuerdo de residencia que le permitió posicionarse como líder en la región en la gobernanza de las migraciones (Margheritis, 2012).

Otros de los avances en el acceso a derechos son los acuerdos multilaterales de Seguridad Social (2006) y Residencia MERCOSUR ampliado (2009), dos ejemplos del acervo de luchas ciudadanas y laborales por incluir mecanismos transnacionales ${ }^{5}$ de protección de los trabajadores (Mondol López, 2010).

El tratamiento dado a los pasos fronterizos es otro de los elementos que nos permiten reflexionar sobre las fronteras nacionales en los procesos de integración. A fines de la década de 1990 se firman varios acuerdos ${ }^{6}$ que apuntan a facilitar y agilizar la movilidad de nacionales y residentes entre localidades de frontera mediante la creación de una Credencial de Tránsito Vecinal Fronterizo (TVF). Asimismo, se redacta el Acuerdo de Recife ${ }^{7}$, a través del cual se busca aunar esfuerzos entre los Estados con el objetivo de avanzar hacia un control unificado de las entradas y salidas de migrantes que se trasladan entre los países de la región. Para ello se establece una noción de control integrado de fronteras, apuntando al desarrollo de procedimientos administrativos y operativos compatibles y similares en forma secuencial y, siempre que sea posible, simultánea, entre los Estados y organismos nacionales para cooperar entre sí, en el ejercicio de los controles aduaneros, migratorios, sanitarios y de transporte.

Un dato importante que evidencia las transformaciones en la manera de entender las migraciones desde el punto de vista regional son las declaraciones en conjunto que, tanto los miembros de MERCOSUR como de UNASUR realizaron a causa de la directiva de retorno ${ }^{8}$, aprobada por la Unión Europea en junio de 2008. El MERCOSUR se reunió en la provincia de Tucumán en julio de 2008, e hizo una declaración respecto de la normativa de retorno, donde se expresa que "deplora su aprobación por considerarla contraria a la propia normativa europea de derechos humanos y a la carta fundamental de la Unión Europea. Además, atenta contra la Convención de los Derechos del Niño, el principio de reunificación familiar y el Protocolo del Estatuto del

5 Relacionado con lo laboral, en la esfera del Grupo Mercado Común se creó el Subgrupo de Trabajo N. 11 dedicado a los Asuntos Laborales en 1991. Al año siguiente una nueva Resolución modifica la denominación del Subgrupo, el cual pasa a llamarse de "Relaciones Laborales, Empleo y Seguridad Social". Este Subgrupo, que funcionó entre 1992 y 1995, tenía como objetivo trabajar el tema de la libre circulación de trabajadores y la seguridad social dentro de la Comisión N. 3 Empleo y Migraciones Laborales (Aguirre, et al, 2007).

6 Decisión CMC 17/99, 18/99, 19/99, 14/00, 15/00.

7 Acuerdo de Recife para la Aplicación de los Controles Integrados de Fronteras entre los Países del MERCOSUR (CMC 05/93).

$8 \quad$ Este documento, establece procedimientos y normas comunes en los Estados miembros de la Unión Europea para ser aplicados con respecto al retorno de nacionales de terceros países que se hallen en situación “ilegal” en su territorio (Pazos, 2009). 
Refugiado" (Nejamkis, 2012, p.5). Por su parte, la UNASUR (2009b, p.1), también expresó su rechazo a "cualquier intento de criminalización, subrayando que los derechos humanos están garantizados por diversos instrumentos internacionales suscritos por países de ambas regiones", resalta además "la importancia de un enfoque integral de los derechos humanos", y exige un "trato justo y humanitario y reciprocidad histórica entendiendo que la libre movilidad es un derecho inherente al ser humano y que la libre circulación debe estar contemplada en el plano internacional".

Acciones como estas vuelven a dar cuenta de las modificaciones tanto en las construcciones como en las miradas en torno al fenómeno migratorio que los bloques regionales, ya sea el MERCOSUR o la UNASUR, tienen sobre las migraciones: en el MERCOSUR ya se trataron los principales lineamientos en materia migratoria que están estrechamente relacionados con las modificaciones en los últimos años de las líneas político-ideológicas de los gobiernos que lo componen; en tanto, la UNASUR implica diversos desafíos, porque representa un espacio nuevo de integración con una cantidad superior de países que lo forman y una mayor heterogeneidad en sus gobiernos.

Por último, nos interesa reflexionar sobre el avance de la construcción de una ciudadanía regional o comunitaria. La promoción de la noción de libre circulación en el plano de la integración contiene ciertos compromisos que propician la conformación de una idea de ciudadanía comunitaria. Sin embargo, queda claro que simplemente con el progreso en esta dirección no se llega a la constitución de una ciudadanía regional.

Para el MERCOSUR, el ejemplo más acabado de promoción de una ciudadanía transfronteriza para habitantes de la región, es el Plan de Acción para el Establecimiento de una Ciudadanía Comunitaria, establecido por el Consejo del Mercado Común (CMC), en su reunión de Foz do Iguaçu de diciembre de 2010 (CMC 64/10). La entrada en vigor de este proyecto se prevé para el año 2021, está integrado por un conjunto de derechos fundamentales y beneficios para los nacionales de los Estados que conforman el Mercosur, e incluye la implementación de una política de libre circulación de personas en la región, igualdad de derechos y libertades civiles, sociales y culturales.

Con una historia más reciente, UNASUR, en la Declaración de Presidentes en la III Reunión Ordinaria del Consejo de Jefas y Jefes de Estado y de Gobierno de la Unión de Naciones Suramericanas, realizada en Quito en agosto de 2009, (UNASUR, 2009a) expresa que:

Reafirman el compromiso de avanzar en la construcción de la ciudadanía suramericana, abordando el tema de la migración con un enfoque integral y comprensivo, bajo el respeto irrestricto de los Derechos Humanos de los migrantes y sus familias, conforme lo dispuesto en la Declaración de Cochabamba, de diciembre de 2006. Para ello, es 
necesario reforzar la cooperación y coordinación regional entre los estados miembros de la UNASUR y la Conferencia Sudamericana de Migraciones, a fin de construir un enfoque común regional que facilite la circulación de personas.

Este espacio político multilateral, cuya formación responde en parte a los compromisos asumidos por los gobiernos en la Conferencia Mundial de Población de El Cairo (1994), es considerado también como un intento de consolidación, ampliación y programación sistemática de los desarrollos alcanzados en los procesos de integración subregional en América del Sur, tanto en la CAN como en el Mercosur (Domenech, 2013).

En esta dirección, y a través de la Declaración de Buenos Aires de mayo de 2010,UNASUR establece en su punto 23 que confirma su interés prioritario de impulsar el proceso de integración en los aspectos más urgentes para el desarrollo humano de las poblaciones, particularmente en lo concerniente al tema migratorio. Para lo cual, inspirada en los principios y objetivos del Tratado Constitutivo, en la Declaración Presidencial de Quito de 10 de Agosto de 2009, así como en los acuerdos alcanzados en la IX Conferencia Suramericana sobre Migraciones, singularmente en lo referente a la construcción de la ciudadanía suramericana, instruye al Consejo de Delegadas y Delegados de la UNASUR, en coordinación con la Conferencia Suramericana, iniciar el proceso de relacionamiento con dicha Conferencia para convenir acciones conjuntas. Esta medida política se ve plasmada en la decisión UNASUR 8/12, en la cual se acuerda en su artículo 1:

Iniciar la construcción de la Ciudadanía Suramericana a partir de su dimensión migratoria, considerando la ampliación de los acuerdos regionales, subregionales y bilaterales, sin perjuicio de los avances que se produzcan en otras dimensiones prioritarias que sobre el tema se estimen prioritarias. (UNASUR, 2012).

Tal como expresa Ramirez (2016), la discusión en torno a esta temática se coloca en dos ejes centrales: por un lado, se plantea reconocer la categoría de ciudadanía suramericana, como una categoría jurídico-política (acceso progresivo a derechos y deberes); por otro, como una categoría socio-cultural asociada a la identidad, tradiciones, costumbres, cosmovisiones entre otras cosas

En relación con los avances, se entiende la propuesta de la ciudadanía comunitaria como el elemento de políticas públicas más acabado en lo que respecta a la regulación de la circulación de personas y el acceso a derechos en los procesos de integración regional. Si bien lo anterior se ha evidenciado en la práctica, ya mediante procesos supranacionales (la Unión Europea), o interestatales (MERCOSUR), que los Estados Nacionales siguen siendo la instancia última de toma de decisiones. En este sentido, su puesta en práctica se expresa como un gran desafío para nuestra región. 


\section{Conclusiones}

Los procesos de integración regional han evidenciado transformaciones en lo que respecta al tratamiento de circulación de personas en la región suramericana. Dos elementos fundamentales intervinieron -especialmente entre los años 2000-2014- en la toma de decisiones de los casos analizados. Por un lado, importantes cambios en la manera de entender la política exterior, ya no basada en un neoliberalismo acérrimo, sino altamente influenciada por una mirada latinoamericanista. Por otro lado, una fuerte intervención de los organismos internacionales, entre los que se destaca como un actor fundamental la Organización Internacional para las Migraciones y su intento permanente de homogeneizar el tratamiento de las migraciones con base en el control.

A su vez, se pudo observar que, en la región, las asimetrías nacionales y subnacionales en materia de niveles y condiciones de vida plantean grandes desafíos al movimiento de personas y a las políticas migratorias. Si bien a nivel de política exterior se avanzó considerablemente en la puesta en común de una política regional de migraciones, se entiende que no todos los países reaccionan de la misma manera ante la recepción de inmigrantes en su territorio, así como tampoco con la emigración de sus connacionales. En este sentido, muchos países no han adoptado aún nuevas leyes de inmigración que se correspondan con el discurso, e incluso la implementación del Acuerdo de Residencia Mercosur, que posibilita el libre movimiento y la igualdad de derechos en la región para la ciudadanía que se traslade de un país a otro, se está llevando a cabo de manera inconsistente y dejando abiertas no pocas preguntas.

Dentro de los principales avances tanto a nivel de MERCOSUR como de UNASUR, se encuentra la propuesta de una ciudadanía comunitaria que todavía no se ha puesto en práctica y en la cual se deberá tener en cuenta el carácter interestatal de los bloques mencionados. No debemos olvidar un componente fundamental que incide directamente en el funcionamiento de la normativa: las instituciones. A lo largo de los años, la puesta en práctica vinculada con las diferentes burocracias de los países se presenta como uno de los principales desafíos al momento de pensar la efectivización de políticas como puede ser la ciudadanía comunitaria.

En relación con lo anterior, el rumbo incierto que ha tomado la región en materia de integración nos posiciona de cara al futuro con más interrogantes que respuestas en relación con el tratamiento de la movilidad de personas y el cumplimiento de los acuerdos ya signados. 


\section{Referencias bibliográficas}

Aguirre, O., Mera, G. y Nejamkis, L. (2007). Migrantes y ciudadanos. Avances y contradicciones del MERCOSUR. En XXVI Congreso de la Asociación Latinoamericana de Sociología (ALAS), Guadalajara, México.

Arcarazo, D., Freier, L. (2015). Discursos y políticas de inmigración en Sudamérica: ¿Hacia un nuevo paradigma o la confirmación de una retórica sin contenido? REMHU: Revista Interdisciplinar da Mobilidade Humana , 23(44), 171-189 Recuperado de https://dx.doi.org/10.1590/198085852503880004411 .

Bernal- Meza, R. (2000). Sistema Mundial y MERCOSUR, Buenos Aires, GEL.

Bono, L., Bogado Bordazar, I. (2014). El MERCOSUR y la UNASUR: ¿Dos experiencias de integración con objetivos convergentes? Conferencia FLACSO-ISA, realizada en Buenos Aires del 23 al 25 de julio de 2014. Centro de Estudios Sudamericanos. Recuperada de http://www.iri.edu.ar/ images/Documentos/CENSUD/boletines/46/art_bono_bogado.pdf.

Carrera, J. (2005). El dilema del MERCOSUR. Buenos Aires, Capital Intelectual.

De Sierra, G. (2001). Los rostros del MERCOSUR. El difícil camino de lo comercial a lo societal. Buenos Aires: CLACSO.

Di Filippo, A. (2000). Globalización, integración regional y migraciones en Di Filippo A y Franco, R. Integración Regional, Desarrollo y Equidad; México, CEPAL/Siglo XXI Editores.

Domenech, E. (2013). Las migraciones son como el agua: Hacia la instauración de políticas de"control con rostro humano". Polis [En línea], 35. Recuperado de http://polis.revues.org/9280 ; DOI : 10.4000/polis.9280.

Domenech, E. (2007). La agenda política sobre migraciones en América del sur:El caso de la Argentina. Revue Européenne des Migrations Internacionales, 23, 2007, 71-94.

Estupiñán Serrano, M. (2013). Acerca de la gobernanza y la gestión migratoria. Crítica del papel de la OIM en el gobierno de la migración internacional y regional (Serie Documentos de Trabajo, Red de Posgrados, 33). Buenos Aires: CLACSO Recuperado de http://biblioteca.clacso.edu. ar/clacso/posgrados/20131118040902/Serrano.pdf. 
García Gutiérrez, A. L. (2002). Tratamiento y análisis de la documentación. En D. Vizcaya Alonso (Comp.), Selección de lecturas: Fundamentos de la organización de la información. La Habana: Universidad de La Habana.

Grimson, A. (2006). Nuevas xenofobias, nuevas políticas étnicas en la Argentina. En A. Grimson, y E. Jellin (Eds.), Migraciones regionales hacia la Argentina, diferencia, desigualdad y derechos. Buenos Aires: Prometeo.

Grimson, A. (2005). Fronteras, Estados e identificaciones en el Cono Sur. En D. Mato (Ed.), Cultura, política y sociedad. Perspectivas latinoamericanas (pp. 127-142). Buenos Aires, Argentina: CLACSO. Recuperado de http://bibliotecavirtual.clacso.org.ar/ar/libros/grupos/mato/ Grimson.rtf.

Iglesias, M., Gomez, A. (2004). Análisis documental y de información: Dos componentes de un mismo proceso. Recuperado de http://www.ucm.es/info/multidoc/verano/material/Felix.htm.

López Sala, A. (2005). Inmigrantes y Estados: La respuesta política ante la cuestión migratoria. Barcelona: Anthropos.

Maguid, A. (2007). Los movimientos migratorios: Determinantes y consecuencias. Tipos de migrantes y fuentes de datos (Documento interno de la Especialización y Maestría en Demografía Social. Universidad Nacional de Luján.

Maguid, A. (1999). Migración e integración en el cono sur: La coexistencia de patrones intra y extrarregionales. En V Jornadas Argentinas de Estudios de Población. Luján, Provincia de Buenos Aires.

Margheritis, A. (2012). Piecemeal regional integration in the post-neoliberal era: Negotiating migration policies within MERCOSUR. Review of International Political Economy, 20(3), 541575.

Mármora, L. (2010). Modelos de gobernabilidad migratoria. La perspectiva política en América del Sur. Revista Interdisciplinar da Mobilidade Humana, REMHU, 18(35), 71-92.

Mármora, L. (2003). Políticas migratorias consensuadas en América Latina. Estudios Migratorios Latinoamericanos, Año 17 № 50.

Mármora, L. (2003). Políticas migratorias consensuadas en América Latina. Estudios Migratorios Latinoamericanos, 17, (50). 
Mármora, L.y PerezVichich, N. (1997). Elementos de políticas migratorias para el Mercosur. En Informe Nacional de Desarrollo Humano. Honorable Senado de la Nación Argentina.

Martínez Pizarro, J. (2011). Migración internacional en América Latina y el Caribe Nuevas tendencias, nuevos enfoques. Santiago de Chile: Comisión Económica para América Latina y el Caribe (CEPAL).

Mellado, N. B. (2009). La integración latinoamericana: Entre interrogantes y teorías. En N. B. Mellado (Ed.), MERCOSUR y UNASUR. ¿Hacia dónde van? (Córdoba: Lerner Ediciones.

Meyer, E. (2006).“Teoría de las políticas migratorias. Un análisis comparado"en:Zona Abierta, 116/117.

Mezzadra, S. (2012). Capitalismo, migraciones y luchas sociales. La mirada de la autonomía. Revista Nueva Sociedad, 237, 159-178.

Modol López, L. (2010). Políticas públicas migratorias: Consideraciones preliminares para su discusión. En Estado actual y perspectivas de las políticas migratorias en el MERCOSUR (pp. 17-13). Montevideo: Flacso - UNESCO.

Nejamkis, L. (2016). Políticas migratorias en Argentina 1976-2010: De la 'Doctrina de Seguridad Nacional' a la consolidación del Derecho Humano a la migración'. Buenos Aires, Argentina: Prometeo.

Nejamkis, L. (2012). Políticas migratorias en tiempos kirchneristas (2003-2010): ¿Un cambio de paradigma? En Migraciones y políticas públicas, nuevos escenarios y desafíos. Buenos Aires: Catálogos-Universidad de Buenos Aires.

Nicolao, J. (2015). Las migraciones en la agenda del MERCOSUR. El rol de Argentina en el Foro Especializado Migratorio. Revista Electrónica de Estudios Internacionales, 29, 1-32.

Novick, S. (2012). Migración y políticas públicas. En S. Novick (Dra.), Nuevos escenarios y desafíos. Buenos Aires: Catálogos.

Novick, S. (2010). Políticas migratorias en la Argentina: Experiencias del pasado, reformas actuales y expectativas futuras. En Estado actual y perspectivas de las políticas migratorias en el MERCOSUR. Montevideo: Flacso - UNESCO.

Novick, S. (2005). La reciente política migratoria argentina en el contexto del Mercosur. En S. Novick, A. Hener, P. Dalle (Eds.), El proceso de integración Mercosur: De las políticas migratorias y de seguridad a las trayectorias de los inmigrantes (Documento de Trabajo 46). Instituto de 
Investigaciones Gino Germani, Facultad de Ciencias Sociales, Universidad de Buenos Aires. Recuperado de http://www.iigg.fsoc.uba.ar/pobmigra/

Pazos, N. (2009). Emigración y políticas de vinculación en Argentina y Uruguay. En VII Encontro Internacional Fórum Universitário MERCOSUL, Rio de Janeiro.

Pérez Vichich, N. (2000). Fundamentos teóricos del tratamiento de la movilidad de personas en el MERCOSUR. En Centro Argentino de Estudios Internacionales, Programa Integración Regional.

Ramírez, G. J. y Alfaro, A. Y. (2010). Espacios multilaterales de diálogo migratorio: El Proceso Puebla y la Conferencia Sudamericana de Migración. Andina Migrante, 9, 2-10.

Ramírez, J. (2016). Hacia el Sur. La construcción de la ciudadanía suramericana y la movilidad intrarregional. Quito: CELAG.

Rhi Sausi, J. L y Conato, D. (2009). Cooperación transfronteriza e integración en américa latina. Roma: Centro Studi di Politica Internazionale e Istituto Italo-Latino Americano.

Sader, E. (2008). Refundar el Estado. Posneoliberalismo en América Latina. Recuperado de http:// bibliotecavirtual.clacso.org.ar/ar/libros/coedicion/sader/sader.pdf.

UNASUR (2012). UNASUR/CJEG/DECISIÓN/No 8 /2012. Recuperado de http://www.unasursg.org/.

UNASUR (2009a). Declaración de Presidentes en la III Reunión Ordinaria del Consejo de Jefas y Jefes de Estado y de Gobierno de la Unión de Naciones Suramericanas Recuperado de http:// www.comunidadandina.org/unasur/10-8-09Dec_quito.htm.

UNASUR (2009b). Declaración de la UNASUR frente a la Propuesta de Directiva de la Unión Europea para sancionar a empleadores de inmigrantes irregulares. Recuperado de http://www. pptunasur.com/documentos.php?menu=5\&idiom=1.

Walters, W. and Haahr, J. H. (2005). Governmentality and Political Studies. European Political Science, $4(3), 288-300$. 